\title{
COMPARAÇÃO DA ESPECIFICIDADE DE LIPASES DE DUAS CEPAS DE Aspergillus niger NA HIDRÓLISE ENZIMÁTICA DO ÓLEO DE GIRASSOL
}

\section{Filipe C.A. Sousa ${ }^{1}$, Erika F. Souza ${ }^{2}$, Selma C. Terzi ${ }^{2}$, Ana Iraidy S. Brigida², Edmar M. Penha $^{2}$ e Leda M.F. Gottschalk ${ }^{2}$}

\author{
${ }^{1}$ Centro Universitário Estadual da Zona Oeste. Av. Manuel Caldeira de Alvarenga 1203, 23.070-200, Rio de \\ Janeiro. \\ 2 Embrapa Agroindústria de Alimentos. Av. das Américas 29501, 23.020-470, Rio de Janeiro, Brasil.
}

E-mail para contato: leda.fortes@embrapa.br

\section{RESUMO}

Diversas pesquisas têm estudado a tecnologia de modificação dos óleos e gorduras e a obtenção de concentrados de ácidos graxos poli-insaturados que beneficiem a saúde do ser humano. As lipases são enzimas que catalisam a hidrólise parcial ou total destes triacilgliceróis (TAGs) produzindo ácidos graxos livres (AGLs), diacilglicerol (DAG), monoacilglicerol (MAG) e glicerol. Neste trabalho, as enzimas produzidas pela cepa mutante Aspergillus niger $11753 A 14$ e pela cepa selvagem Aspergillus niger $C$ foram utilizadas para a hidrólise do óleo de girassol. As lipases foram caracterizadas quanto à sua espeficidade em relação a diferentes p-nitrofenil ésteres e aplicadas na hidrólise de óleo de girassol. As condições da hidrólise enzimática do óleo de girassol foram: proporção molar de água:óleo (8:1); carga da lipase de $5 \mathrm{U} / g$ de óleo; $50^{\circ} \mathrm{C}$ e agitação orbital de $150 \mathrm{rpm}$. Os ácidos graxos livres foram quantificados em titulador automático com NaOH 0,05 N. Os melhores resultados de hidrólise foram obtidos com extrato bruto de A. niger C, que apresentaram $228 \mathrm{mM}$ de ácidos graxos livres em 5,5 horas de reação.

\section{INTRODUÇÃO}

A importância dos ácidos graxos poli-insaturados (AGPIs) essenciais, como os ácidos linoléico e $\alpha$ linolênico na dieta humana tem sido assunto de grande relevância (Kraus-Etshcmann et al., 2007; Feltes et al., 2013). Esses AGPIs são essenciais, pois não podem ser sintetizados pelo organismo 


\section{SIMPÓSIO NACIONAL DE BIOPROCESSOS \\ XI SIMPÓSIO DE HIDRÓLISE ENZIMÁTICA DE BIOMASSA}

01 a 04 de setembro de 2015

Fortaleza, Ceará, Brasil

humano devendo ser fornecidos através da dieta. O concentrado de AGPIs possui uma ampla aplicação na indústria de alimentos, sendo fonte de ácidos graxos específicos e desejáveis para fins nutricionais. Diversos estudos têm demonstrado o uso de lipases na modificação de óleos e gorduras, como na obtenção de AGPIs e de lipídeos estruturados (Munio et al., 2009; Nunes et al., 2011).

As lipases são enzimas hidrolíticas, que em ambientes aquo-restritos são capazes de catalisar diversas reações como esterificação, transesterificação e interesterificação (Sharma et al., 2001). Para decidir sua aplicação final, primeiramente é necessário conhecer a especificidade das lipases. As lipases microbianas podem ser classificadas em três grupos: específica em relação à posição 1,3 do ácido graxo no triglicerídeo, não-específica ou específica em relação ao tamanho da cadeia do ácido graxo. As lipases ácido graxo específicas são aquelas que catalisam a hidrólise de tipos específicos de grupos acilas nas moléculas de TAG.

O objetivo deste trabalho foi produzir e avaliar a especificidade de dois extratos enzimáticos brutos produzidos por duas cepas de Aspergillus niger, além de utilizá-los na obtenção de concentrados de ácidos graxos poli-insaturados por hidrólise enzimática do óleo de girassol.

\section{MATERIAIS E MÉTODOS}

\subsection{Produção de Lipase por Fermentação em Estado Sólido}

O extrato enzimático lipolítico foi produzido através de fermentação em estado sólido pelos fungos Aspergillus niger 11T53A14, cepa geneticamente modificada por métodos clássicos, e Aspergillus niger $C$, isolada em amostras de margarina, ambas da Coleção de Microrganismos de Interesse da Indústria de Alimentos e Agroenergia, da Embrapa Agroindústria de Alimentos. Os experimentos foram conduzidos em colunas aeradas, incubadas em banho-maria a $32{ }^{\circ} \mathrm{C}$ e com entrada controlada de ar não umedecido de 1,0 vvm por 96 horas. Os fungos foram inoculados em cada coluna contendo $40 \mathrm{~g}$ de meio (torta de dendê umidificada com $80 \mathrm{~mL}$ de solução de sulfato de amônio e com adição de $3 \%$ de borra de dendê como indutor). A enzima foi extraída com a adição de $2,5 \mathrm{~mL}$ de tampão fosfato de sódio $0,1 \mathrm{M}(\mathrm{pH} 7,0)$ por grama de meio fermentado, permanecendo por 1 hora sob agitação de $90 \mathrm{rpm}$ por $1 \mathrm{~h}$ em shaker com banho a $32{ }^{\circ} \mathrm{C}$. $\mathrm{O}$ extrato enzimático bruto foi obtido após filtração com papel de filtro seguido de filtração em membrana de microfiltração $(0,45 \mu \mathrm{m})$ para posterior determinação das atividades enzimáticas.

\subsection{Determinação da Atividade}

A atividade lipásica expressa o potencial catalítico da enzima na hidrólise do óleo presente na emulsão (água-óleo de oliva-goma arábica), liberando ácidos graxos e glicerol. A determinação da atividade lipásica no extrato bruto foi realizada por método titulométrico, segundo o procedimento descrito por Pereira et al. (2001), com pequenas modificações: adicionou-se $1 \mathrm{~mL}$ do extrato enzimático a $4 \mathrm{~mL}$ de tampão citrato de sódio $50 \mathrm{mM} \mathrm{pH} \mathrm{4,0} \mathrm{e} 5 \mathrm{~mL}$ de emulsão de goma arábica (48 $\mathrm{mL}$ de água destilada, $48 \mathrm{~mL}$ de óleo de oliva e $7 \mathrm{~g}$ de goma arábica). Após $15 \mathrm{~min}$ a $35^{\circ} \mathrm{C}$, a reação foi interrompida com a adição de $10 \mathrm{~mL}$ de uma solução 1:1:1 de acetona/etanol/água e a 


\section{SIMPÓSIO NACIONAL DE BIOPROCESSOS \\ XI SIMPÓSIO DE HIDRÓLISE ENZIMÁTICA DE BIOMASSA}

01 a 04 de setembro de 2015

Fortaleza, Ceará, Brasil

titulação realizada com $\mathrm{NaOH}(0,05 \mathrm{~N})$, até pH final 11,0, e o resultado foi expresso em $\mathrm{U}$ por grama de substrato seco (SS) utilizado como meio para a produção da enzima (U.g $\mathrm{gss}^{-1}$ ). O branco da reação foi realizado adicionando-se a enzima no momento da titulação. Uma unidade de atividade lipásica (U) foi definida como a quantidade de enzima capaz de produzir $1 \mu \mathrm{mol}$ de ácidos graxos (oleico) por minuto, sob as condições de ensaio padrão. Toda a análise foi feita em duplicata para os brancos e em triplicata para as amostras.

\subsection{Avaliação da Especificidade da Lipase}

Avaliação das especificidades das lipases de Aspergillus niger (C e 11T53A14) foi realizada através da hidrólise de $\mathrm{p}$-nitrofenil ésteres com cadeias de tamanho curto (acetato, C2; butirato, C4), médio (octanoato, C8; laurato, C12) e longo (palmitato, 16). Todos os substratos e o padrão de pnitrofenol utilizados para este teste foram adquiridos da Sigma Chemical Co. (St. Louis, EUA). Para tanto, uma solução de extrato enzimático foi preparada em tampão fosfato $50 \mathrm{mM} \mathrm{pH} \mathrm{7,0.} \mathrm{Os}$ diferentes substratos foram solubilizados em álcool isopropílico e/ou DMSO e depois homogeneizados em tampão fosfato $50 \mathrm{mM} \mathrm{pH} \mathrm{7,0,} \mathrm{de} \mathrm{forma} \mathrm{que} \mathrm{a} \mathrm{concentração} \mathrm{dos} \mathrm{substratos}$ foi de $560 \mu \mathrm{M}$. A reação foi acompanhada pela liberação de p-nitrofenol no meio reacional, o qual foi detectado por leitura espectrofotométrica a $410 \mathrm{~nm}$ (Stoytcheva et al., 2012). Em tubo de ensaio, $2 \mathrm{~mL}$ do substrato foram aclimatados a 37으 $\mathrm{Cm}$ banho-maria. A seguir $100 \mu \mathrm{L}$ do preparado enzimático foram adicionados ao tubo de ensaio e, em seguida, transferidos para a cubeta para acompanhamento do aumento da absorvância a $410 \mathrm{~nm}$ por 100 segundos. Todas as análises foram feitas em triplicata. A atividade enzimática foi calculada de acordo com a Equação 1 e graficamente expressa em percentual relativo a atividade mais alta de cada enzima para cada substrato.

$A=[(\Delta A b s / \Delta t) . f . V r] / V a$

Onde:

A - atividade da enzima (U/L), onde uma unidade (U) de atividade enzimática hidrolítica é definida como a quantidade de enzima que libera $1 \mu \mathrm{mol}$ de p-nitrofenol por minuto nas condições do ensaio;

$\Delta$ Abs - variação de absorbância no intervalo de tempo ( $\Delta \mathrm{t}$, em minutos) transcorrido durante a fase de aumento linear da absorbância;

$\Delta \mathrm{t}$ - o tempo decorrido de análise, em minutos;

$V r$ - volume reacional total (L);

$V a$ - volume da solução amostra (L);

$f$ - fator de conversão dos valores de absorvância para a concentração de p-nitrofenol (109,3 $\mu \mathrm{mol} / \mathrm{L})$. 


\section{SIMPÓSIO NACIONAL DE BIOPROCESSOS \\ XI SIMPÓSIO DE HIDRÓLISE ENZIMÁTICA DE BIOMASSA}

\subsection{Hidrólise Enzimática do Óleo de Girassol}

A hidrólise do óleo de girassol foi realizada em duplicata utilizando uma razão molar de óleo:água de 1:8. A carga de enzima aplicada foi padronizada para $5 \mathrm{U}$ por grama de óleo. Um branco reacional foi realizado adicionando apenas água juntamente com o óleo de girassol. A reação ocorreu sob agitação de $150 \mathrm{rpm}$ e $50{ }^{\circ} \mathrm{C}$. Amostras do meio reacional ( $1 \mathrm{~mL}$ ) foram coletadas a 0,5 , $1,1,5,2,3$, 4 e 5 horas de reação. A reações foram interrompidas com $1 \mathrm{~mL}$ de reagente de parada (Água, Acetona, Etanol (1:1:1)) e foi adicionado $28 \mathrm{~mL}$ de água destilada para equilibrar o volume. A concentração de ácidos graxos livres foi determinada por titulação com $\mathrm{NaOH} 0,05 \mathrm{~N}$.

\section{RESULTADOS}

Os resultados de produção da lipase pelas duas cepas mostraram que o fungo A. niger 11T53A14 atingiu valor máximo (72,57 U.gss-1, em 48 h de fermentação) muito próximo ao do $A$. niger $C$ (67,49 U.gss-1, em 96 h de fermentação). Embora os valores da atividade enzimática estejam próximos, o tempo de fermentação para alcançá-los foi maior para a cepa A. niger C (Figura 1).

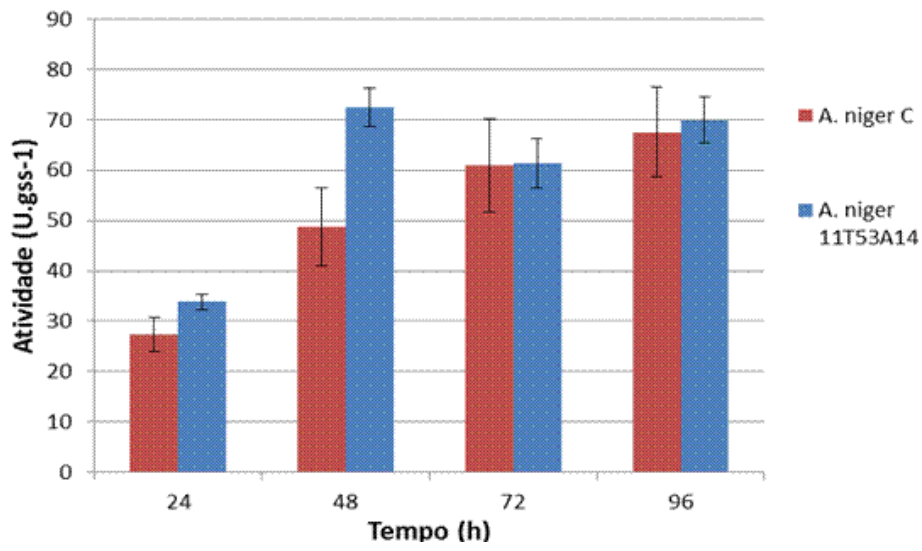

Figura 1. Comparação da produção de lipase por A.niger 11T53A14 e A.niger C em torta de dendê umidificada com solução de sulfato de amônio e com indutor de borra de dendê.

Em relação à especificidade dos extratos brutos produzidos (Figura 2), $A$. niger $C$ produziu um extrato com alta especificidade para substrato com cadeia carboxílica de 8 carbonos, apresentando menor atividade para 12 e 16 carbonos e atividade pouco expressiva para cadeia curta ( 2 e 4 carbonos). O fungo A. niger 11T53A14 produziu um extrato menos específico quando comparado ao fungo $A$. niger $C$. Valores de atividade elevados foram observados para substratos com cadeia carboxílica de 8 e 16 carbonos. Porém, ao contrário do observado para o extrato de $A$. niger $\mathrm{C}$, o extrato de $A$. niger 11 T53A14 apresentou valores de atividade para substratos com cadeia curta (acetato), maior que $50 \%$ do valor máximo observado. 


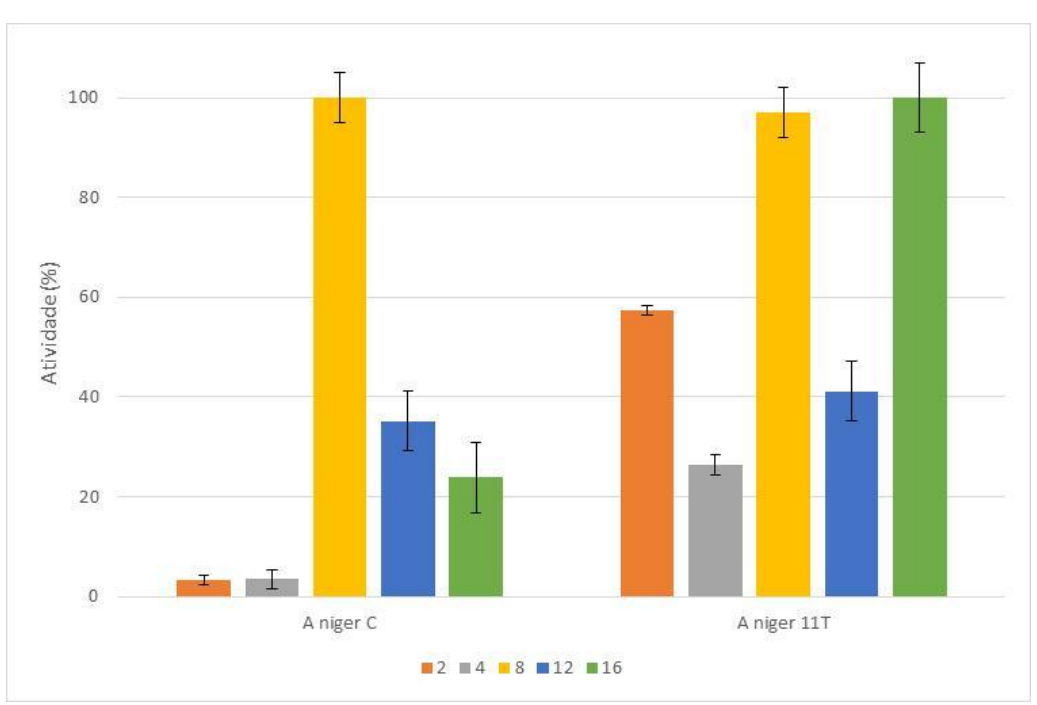

Figura 2. Comparação da especificidade dos extratos produzidos por A. niger 11T53A14 e A. niger C frente a diferentes p-nitrofenil ésteres com cadeia carboxílica de 2, 4, 8, 12 e 16 carbonos.

Os dois extratos brutos obtidos foram utilizados para a hidrólise enzimática do óleo de girassol. Este óleo foi escolhido por possuir cerca de $60 \%$ de ácido linoleico, considerado essencial ao organismo humano. Os melhores resultados foram obtidos, com o extrato obtido com a cepa de $A$. niger $\mathrm{C}$, onde se observou uma concentração de $228 \mathrm{mM}$ de ácido graxo em 5,5 h de reação. Quando o extrato enzimático da cepa A. niger 11T53A14 foi utilizado, apesar deste ser menos específico e apresentar maior atividade para p-nitrofenil palmitato (C16), a hidrólise do óleo de girassol foi praticamente nula. Avaliando uma possível simbiose entre os extratos, misturaram-se os mesmo numa proporção enzimática $(\mathrm{m} / \mathrm{m})$ de 1:1. O aumento foi pouco expressivo frente ao resultado observado para a reação com extrato da cepa $A$. niger $\mathrm{C}$, embora dados da literatura sugiram que o uso concomitante de extratos enzimáticos com especificidade diferentes poderia melhorar a ação da lipase nos diferentes ácidos graxos presentes em óleo vegetais (Rodrigues e Ayub, 2011).

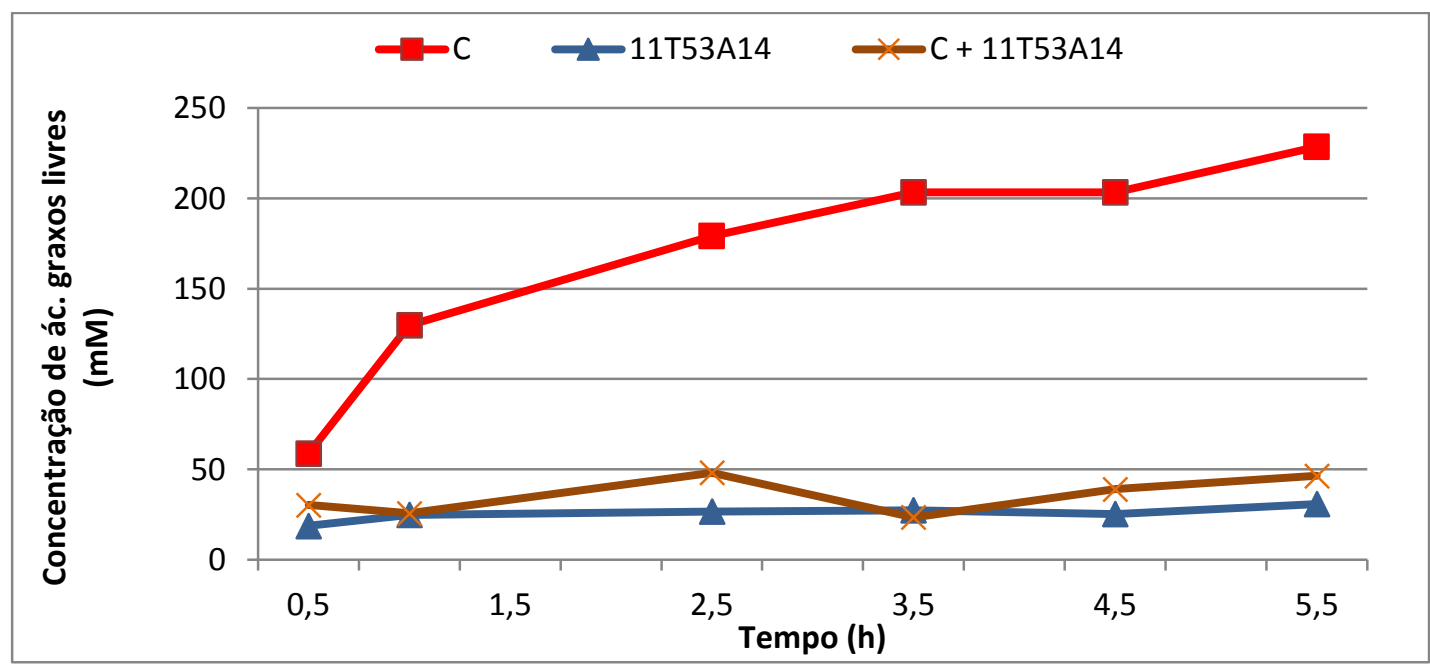

Figura 3. Concentração de ácido graxos livres obtidos durante a hidrólise de óleo de girassol. 


\section{SIMPÓSIO NACIONAL DE BIOPROCESSOS \\ XI SIMPÓSIO DE HIDRÓLISE ENZIMÁTICA DE BIOMASSA}

01 a 04 de setembro de 2015

Fortaleza, Ceará, Brasil

\section{CONCLUSÕES}

Nota-se que apesar do tempo de produção da lipase pela cepa mutante 11T53A14 ser menor e sua especificidade ser mais abrangente na hidrólise de $p$-nitrofenol com cadeias de diferentes tamanhos em comparação com a lipase produzida pela cepa selvagem C, na hidrólise do óleo de girassol os melhores resultados foram obtidos com o extrato enzimático produzido pela cepa selvagem Aspergillus niger C. Quando os extratos enzimáticos foram misturados na mesma proporção, com atividade enzimática próxima, não agiram de forma sinérgica, apresentando rendimento de hidrólise muito baixo.

\section{REFERÊNCIAS}

Feltes, M.M.C., Oliveira, D., Block, J.M., Ninow, J.N., 2013. The production, benefits and applications of monoacylglycerols and diacylglycerols of nutritional interest. Food Bioprocess Technol. 6, 17-35.

Kraus-Etshcmann, S., Shadid, R., Campoy, C., Hoster, E., Demmelmair, H., Jiménez, M., Gil, A., Rivero, M., Veszprémi B., Decsi, T., Koletzko, B. V. 2007. Effects of fish oil and folate supplementation of pregnant women on maternal and fetal plasma concentrations of docosahexaenoic acid and eicosapentaenoic acid: a European randomized multicenter trial. Am J of Clin Nutr. 85, 1392-1400.

Munio, M.M., Robles, A., Esteban, L., Gonzalez, P.A., Molina, E., 2009. Synthesis of structured lipids by two enzymatic steps: ethanolysis of fish oils and esterification of 2-monoacylglycerols. Process Biochem. 44, 723-730.

Nunes, P.A., Pires-Cabral, P., Ferreira-Dias, S., 2011. Production of olive oil enriched with medium chain fatty acids catalyzed by commercial immobilized lipases. Food Chem. 127, 993-998.

Pereira, E.B., Castro, H.F., Moraes, F.F., Zanin, G.M., 2001. Kinetic studies of lipase from Candida rugosa: a comparative study between free and enzyme immobilized onto porous chitosan beads. Appl Biochem Biotechnol. 91-93, 739-752.

Rodrigues, R.C.; Ayub, M.A.Z., 2011. Effects of the combined use of Thermomyces lanuginosus and Rhizomucor miehei lipases for the transesterification and hydrolysis of soybean oil. Process Biochem. 46, 682-688.

Sharma, R., Chisti, Y., Banerjee, U.C., 2001. Production, purification, characterization, and applications of lipases. Biotechnol Adv. 19, 627-662.

Stoytcheva, M., Montero, G., Zlatev, R., Leon, J.A., Gochev, V., 2012. Analytical methods for lipases activity determination: A review. Curr Anal Chem. 8, 400-407. 